\title{
Convectively unstable turbulent open channel flow with stable surface stratification
}

\author{
N. Williamson ${ }^{1}$ \\ M. P. Kirkpatrick ${ }^{2}$ \\ S. W. Armfield ${ }^{3}$ \\ S. E. Norris ${ }^{4}$
}

(Received 18 February 2015; revised 9 November 2015)

\begin{abstract}
We use direct numerical simulations to examine fully developed turbulent open channel flow where the near wall region is unstably stratified and the outer boundary layer is stably stratified. The simulations are a model for flow in shallow turbid river channels with incident solar radiation. The aim is to determine under what conditions and by what mechanism the stably stratified layer is overturned. The flow is attained by applying a radiative heat flux at the free surface of the open channel. The absorption and transmission of the radiation follows the Beer-Lambert law with a constant absorption coefficient. We examine conditions where approximately $20 \%$ of the incident radiative heat flux penetrates through to the wall, releasing heat at the wall as a
\end{abstract}

http://journal.austms.org.au/ojs/index.php/ANZIAMJ/article/view/9312 gives this article, (c) Austral. Mathematical Soc. 2016. Published January 13, 2016, as part of the Proceedings of the 17th Biennial Computational Techniques and Applications Conference. ISSN 1446-8735. (Print two pages per sheet of paper.) Copies of this article must not be made otherwise available on the internet; instead link directly to this URL for this article. 
heat flux. The problem is specified by our buoyancy parameter which is analogous to the bulk Obukhov length scale. In the stable outer boundary layer we observe that the flux Richardson number reaches the limiting value, as was observed in the atmospheric boundary layer under sheared convective conditions.

\section{Contents}

1 Introduction

2 Problem Formulation

2.1 Direct numerical simulations . . . . . . . . . . . . C252

3 Results

C253

4 Conclusions

C259

References

C259

\section{Introduction}

In river systems and in coastal seas, surface heating by incident solar radiation can produce a region of stable density stratification within the water column. The stratified layer opposes vertical fluid motion and suppresses turbulent mixing, and therefore reduces the vertical exchange of nutrients and dissolved oxygen from the surface to the river bed. This in itself is an unwanted environmental outcome. In Australian inland river systems, stratified conditions were also correlated with the onset of algal-blooms [1]. It is desirable to be able to predict the onset of strongly stratified stable conditions so that flow management strategies may be employed. This requires an understanding of 
how turbulence is generated and dissipated within the water column under different environmental conditions.

The dynamics of turbulent stably stratified channel flow were considered in many flow settings $[2,3,4]$. We consider open channel flow where the free surface region is stably stratified and the near bed region is unstably stratified. This flow occurs when a portion of solar radiation penetrates the water column to the river bed. The heat released at the bed can rise as thermal plumes. The potential energy released by the convective plumes may disrupt the stable surface stratification. The flow is analogous to the sheared convective atmospheric boundary layer and we show that many features of that flow are recognisable in the present setting. In this study we use direct numerical simulations to examine the energy transfers within the water column under these conditions.

\section{Problem Formulation}

Our flow arrangement is illustrated in Figure 1.

An incident radiative heat flux $\mathrm{I}_{\mathrm{s}}$ penetrates the top surface and is absorbed within the fluid domain following the Beer-Lambert law to produce a volumetric heat source

$$
\mathrm{q}(z)=\mathrm{I}_{\mathrm{s}} \alpha \mathrm{e}^{-(\delta-z) \alpha},
$$

where $z$ is the vertical distance from the wall (or river bed), $\alpha$ is the absorption coefficient and $\delta$ is the domain height (or channel depth). At $z=0$ the fraction of energy remaining at the wall is $\mathrm{q}_{w}=\mathrm{I}_{\mathrm{s}} \mathrm{e}^{-\alpha \delta}$. In our model, this heat is released as a heat flux at the wall. The flow is periodic in the horizontal plane and driven by a constant pressure gradient in the stream-wise $x$ direction. After the flow attains a statistically steady horizontally homogeneous state, the energy input from the source terms is transported across the channel at a constant rate. 
Figure 1: Schematic of flow. Domain is periodic in the stream-wise $x$ direction and the span-wise $y$ direction. The lower $z$ boundary is a no-slip wall. The shaded area indicates the volumetric heat source.

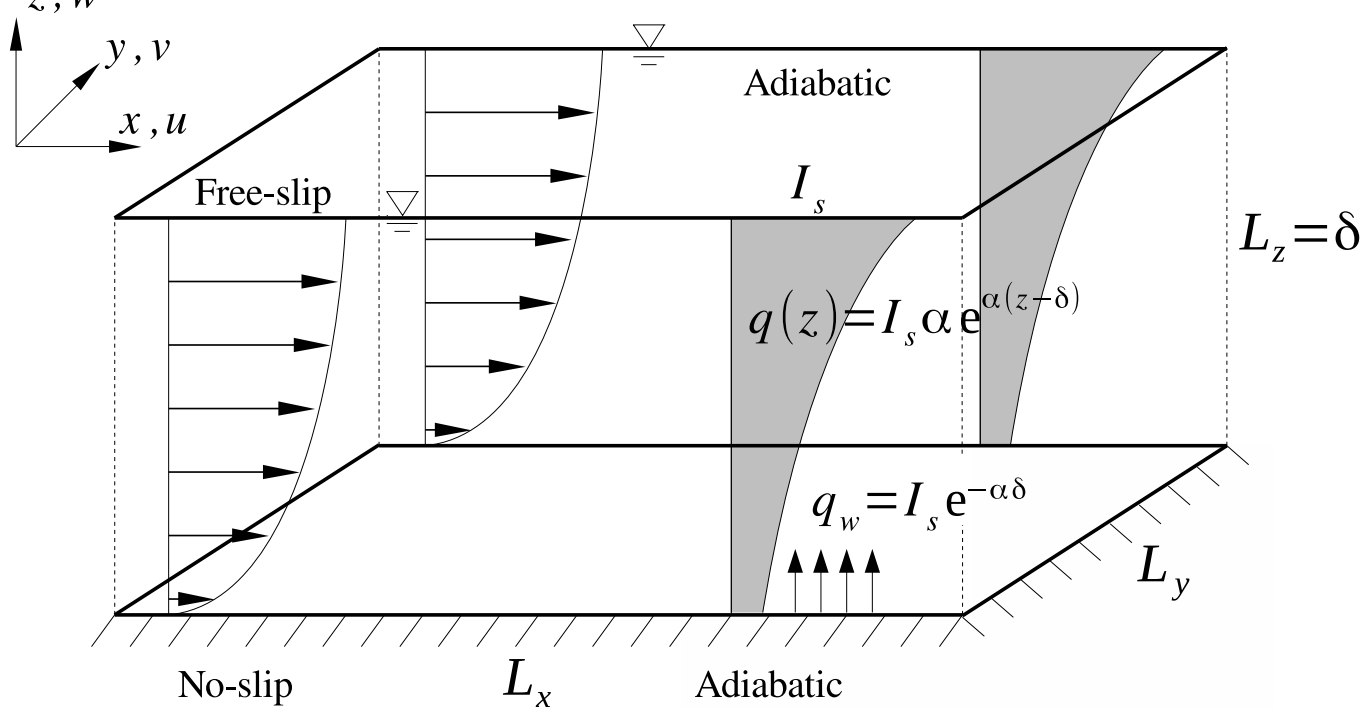

The dimensional temperature field at time $\mathrm{T}$ is decomposed as

$$
\Phi(x, T)=\Phi^{\prime}(x, T)+\bar{\Phi}(T),
$$

where $\Phi^{\prime}$ is the steady temperature field and the uniform increase in temperature with time is

$$
\partial \bar{\Phi} / \partial T=\overline{\mathrm{I}}_{\mathrm{s}} / \rho_{0} \mathrm{C}_{\mathrm{p}},
$$

where $\rho_{0}$ is the fluid density and $C_{p}$ is the fluid specific heat. We obtain a nondimensional statistically steady temperature field $\phi$ and a non-dimensional heat source $\mathrm{q}_{e}(z)$ by normalising with $\Phi_{\mathrm{N}}=\mathrm{I}_{\mathrm{s}} / \rho_{0} \mathrm{C}_{\mathrm{p}} \mathrm{u}_{\tau}$ and $\mathrm{q}_{\mathrm{N}}=\mathrm{I}_{\mathrm{s}} / \delta$, respectively, to give,

$$
\phi=\frac{\Phi-\bar{\Phi}(\mathrm{T})}{\Phi_{\mathrm{N}}}, \quad \mathrm{q}_{e}(z)=\frac{\mathrm{q}(z)-\mathrm{q}_{\mathrm{N}}}{\mathrm{q}_{\mathrm{N}}}
$$


where $u_{\tau}=\sqrt{\tau_{w} / \rho_{0}}$ is the friction velocity and $\tau_{w}$ is the plane averaged shear stress at the wall. With this normalisation we perform direct numerical simulations (DNS) of the Navier-Stokes equations.

We consider an incompressible fluid with the Oberbeck-Boussinesq approximation for buoyancy. The governing equations for the conservation of mass, momentum and energy are written in non-dimensional form as

$$
\begin{aligned}
\nabla \cdot \mathbf{u} & =0 \\
\frac{\partial \mathbf{u}}{\partial \mathrm{t}}+\nabla \cdot(\mathbf{u} \mathbf{u}) & =-\nabla p+\frac{1}{\operatorname{Re}_{\tau}} \nabla^{2} \mathbf{u}+\mathbf{e}_{\mathrm{x}}+\lambda \phi \mathbf{e}_{z}, \\
\frac{\partial \phi}{\partial \mathrm{t}}+\nabla \cdot(\mathbf{u} \phi) & =\frac{1}{\operatorname{Re}_{\tau} \operatorname{Pr}} \nabla^{2} \phi+\mathrm{q}_{\mathrm{e}},
\end{aligned}
$$

where $\boldsymbol{e}_{\mathrm{x}}$ and $\boldsymbol{e}_{z}$ are the unit vectors in the $\mathrm{x}$ and $z$ directions, respectively. The Prandtl number $\operatorname{Pr}=v / \sigma=0.71$ where $\sigma$ is the scalar diffusivity of the fluid and $v$ the fluid viscosity. The Reynolds number $\operatorname{Re}_{\tau}=\mathfrak{u}_{\tau} \delta / v=400$. The velocity vector is $\mathbf{u}=(\mathfrak{u}, v, w)$ along the $x, y$ and $z$ directions, respectively, and the velocity components are normalised by $\mathfrak{u}_{\tau}$. The non-dimensional time $t$ and pressure $p$ are obtained using $t=T u_{\tau} / \delta$ and $p=P / \rho_{0} u_{\tau}^{2}$ where $\mathrm{T}$ and $\mathrm{P}$ are the dimensional time and pressure, respectively. Length scales are normalised by channel depth $\delta$.

The buoyancy parameter $\lambda=\delta / L$ is the ratio of the channel depth $\delta$ to a bulk Obukhov length scale $L=u_{\tau}^{3} \rho_{0} C_{p} / I_{s} g \beta$ where $\beta$ is the coefficient of thermal expansion for the fluid. We vary $\lambda$ over the range 0 to 20. We used a similar approach in an earlier study [4], which provides further details. We obtain a stability parameter for the convective flow at the wall $\lambda_{w}=q_{w} g \beta \delta / u_{\tau}^{3} \rho C_{p} \equiv$ $\lambda e^{-\alpha \delta}$. The strength of the convective heat flux released from the wall is controlled via the absorption coefficient $\alpha \delta$. The governing parameters for this problem are then $\operatorname{Re}_{\tau}, \lambda, \alpha \delta$ and $\operatorname{Pr}$.

The boundary conditions for the bottom $(z=0)$ no-slip adiabatic wall and 
stress free adiabatic top boundary $(z=1)$ are

$$
\begin{array}{ll}
z=0: & u=v=w=0, \quad \frac{\partial \phi}{\partial z}=-\operatorname{Re}_{\tau} \operatorname{Pr} e^{-\alpha \delta}, \\
z=1: & \frac{\partial u}{\partial z}=\frac{\partial v}{\partial z}=w=0, \quad \frac{\partial \phi}{\partial z}=0 .
\end{array}
$$

In the statistically steady flow considered here, the non-dimensional time averaged temperature transport equation obtained from equation (7) is

$$
\frac{d\left\langle\phi^{\prime} w^{\prime}\right\rangle}{d z}=\frac{1}{\operatorname{Re}_{\tau} \operatorname{Pr}} \frac{d^{2} \phi}{d z}+q_{e} .
$$

where $\langle\cdot\rangle$ indicates averaging in the horizontal plane and in time. The primes indicate fluctuations from the mean, so $w^{\prime}=\langle w\rangle-w$. In the large Reynolds number limit, $1 /\left(\operatorname{Re}_{\tau} \operatorname{Pr}\right) \rightarrow 0$, we integrate over the channel height with the boundary condition $\left\langle\phi^{\prime} w^{\prime}\right\rangle=0$ at $z=1$ to obtain the turbulent heat flux profile

$$
-\left\langle\phi^{\prime} w^{\prime}\right\rangle=z-e^{(z-1) \alpha \delta} .
$$

Integrating the buoyancy flux $-\lambda\left\langle\phi^{\prime} w^{\prime}\right\rangle$ over the channel height gives the potential energy required to maintain statistically steady conditions,

$$
-\int_{0}^{1} \lambda\left\langle\phi^{\prime} w^{\prime}\right\rangle d z=\frac{\lambda}{2}-\frac{\lambda}{\alpha \delta}\left(1-e^{-\alpha \delta}\right) .
$$

In this study we examine the case where $\alpha \delta=1.5936$. With this arbitrary choice the integral in (12) is zero so the net potential energy required for steady conditions is approximately zero. In other words, the potential energy released at the wall is the same magnitude as the work required to completely mix down the stable surface layer. This is an arbitrary choice for $\alpha \delta$ which allows us to examine a flow that simultaneously contains both strongly stable and strongly convective regions. Larger or smaller values of $\alpha \delta$ result in more or less stable flow, respectively. 


\subsection{Direct numerical simulations}

Equations (5)-(7) are solved using the fractional step finite volume solver described by Armfield et al. [5]. The code uses a cell-centred co-located storage arrangement for flow variables on a regular structured grid, with cell-face velocities calculated using the Rhie-Chow momentum interpolation. The spatial derivatives are discretised using second order central finite differences. A second-order accurate Adams-Bashforth time advancement scheme is used for the non-linear terms, and a Crank-Nicolson for the time advancement of the diffusive terms. The pressure correction equation is solved using a stabilised bi-conjugate gradient solver with an incomplete Cholesky factorisation preconditioner. The momentum and temperature equations are solved using a Jacobi solver. The computational domain extends in the stream-wise $x$, span-wise $y$ and wall normal directions with lengths $L_{x}=2 \pi, L_{y}=\pi, L_{z}=1$, respectively. The length scale of the smallest turbulent eddies in the flow is the Kolmogorov length scale, which in viscous wall units is $\eta^{+}=\operatorname{Re}_{\tau} \eta / \delta$. Our simulations found the Kolmogorov length scale to range from $\eta^{+}=1.5$ to 5 for $\lambda=20$. The grid size in wall units is $\Delta x^{+}=\operatorname{Re}_{\tau} \Delta x \simeq 5$ with $N_{x}=512$ nodes and $\Delta y^{+} \simeq 2.5$ with $\mathrm{N}_{y}=512$ nodes. In the vertical axis, the grid is stretched from $\Delta z^{+}=0.55$ at the wall to $\Delta z^{+}=1$ at $z=1.0$ with $\mathrm{N}_{z}=130$ nodes. A Courant number limit of between $0.18-0.2$ is used to obtain the time step size.

The simulation for the neutral $\lambda=0$ flow was initialised with a turbulent mean velocity profile and a large random perturbation. After an initial transient phase, typically $\Delta t=40$ (non-dimensional time units $\delta / T u_{\tau}$ ) statistically steady conditions were assumed. The flow was then evolved for a further period, typically $\Delta t=40$, and statistics were collected. Higher $\lambda$ flow conditions were successively initialised from these flow fields and computations continued in the same manner. We present statistically steady flow statistics only. 


\section{Results}

The first conclusion is that the flow structure is remarkably similar to that seen in the sheared convective atmospheric boundary layer (SCBL). The mean temperature profile through the channel depth is shown in Figure 2(a) for $\lambda=0$ to 20. The flow is comprised of a unstable near wall region $z<0.2$, a well mixed layer, $z=0.2$ to 0.5 , and a stably stratified layer for $z \gtrsim 0.5$. The root mean square (rms) temperature fluctuation $\phi_{\mathrm{rms}}$ is a minimum in the mixed layer, as shown in Figure 2(c). The heat flux is shown in Figure 2(b) for $\lambda=4$ to 80 . For $z \lesssim 0.36$ the heat flux $-\left\langle\phi^{\prime} w^{\prime}\right\rangle<0$ indicating turbulence production or convective flow, while for $z \gtrsim 0.36$ the heat flux works against turbulence and acts as a net sink of turbulence. These features are seen in the developing SCBL [6].

We are primarily interested in identifying the onset of the stable flow in the near free surface region in terms of $\lambda$. In neutral conditions $\lambda=0$, the velocity field and the temperature field are decoupled and the flow is turbulent throughout the domain. As buoyancy strength is increased from zero both the convective and stable regions exert influence on the flow structure. The change in flow structure is visualised through the instantaneous temperature contours given in Figure 3. At $\lambda=4$ domain scale eddies penetrate to the surface. At $\lambda=10$ and 20 there is a distinct shear layer below the free surface with overturns in the temperature field observable. At $\lambda=20$ turbulence in the free surface region is increasingly damped. The shear stress $\left\langle u^{\prime} v^{\prime}\right\rangle$ and the stream-wise normal stress $\mathfrak{u}_{\text {rms }}$ are damped over $z>0.8$ compared with $\lambda=0$ flow, as shown in Figure 4(a)-(b). The mean stream-wise velocity profiles are flattened in the convective region of the flow, as shown in Figure 4(c).

The contribution to turbulence production by shear and buoyancy though the domain height is seen through the balance terms in the turbulent kinetic energy (TKE) transport equations. In non-dimensional form the TKE equations are

$$
\mathrm{CT}+\frac{\mathrm{d}}{\mathrm{d} z}\left\langle w^{\prime} p^{\prime}\right\rangle-\frac{1}{\operatorname{Re}_{\tau}} \frac{\mathrm{d}^{2} k}{\mathrm{~d} z^{2}}=\mathrm{SP}-\varepsilon-\mathrm{B},
$$


Figure 2: Variations over the vertical location $z$ of (a) the mean temperature profile relative to the mean temperature at the wall $\phi-$ $\phi_{0}=\phi-\langle\phi\rangle_{z=0}$; (b) the heat flux $-\left\langle\phi^{\prime} w^{\prime}\right\rangle$; and (c) the rms temperature fluctuation $\phi_{\text {rms }}$.

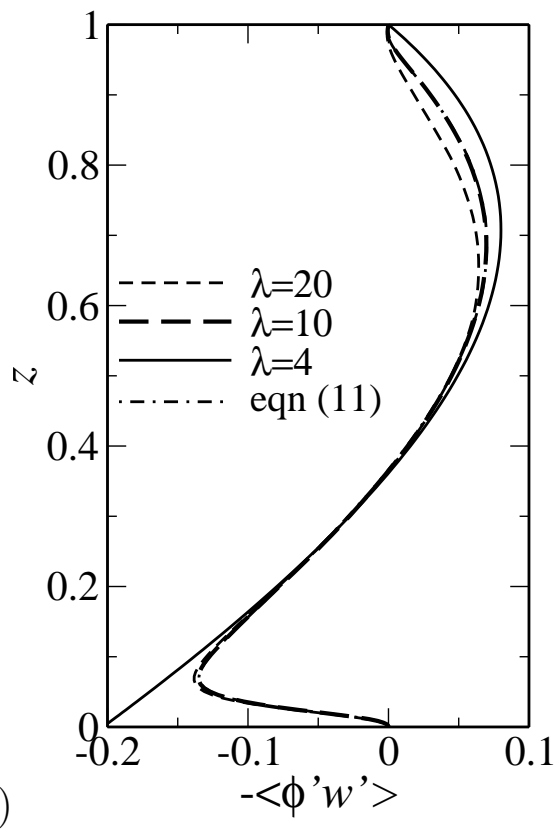

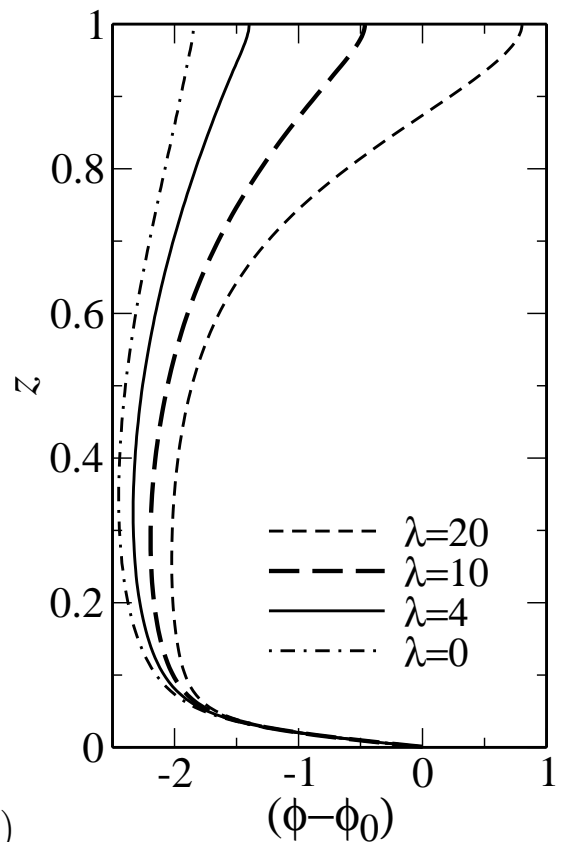

(a)

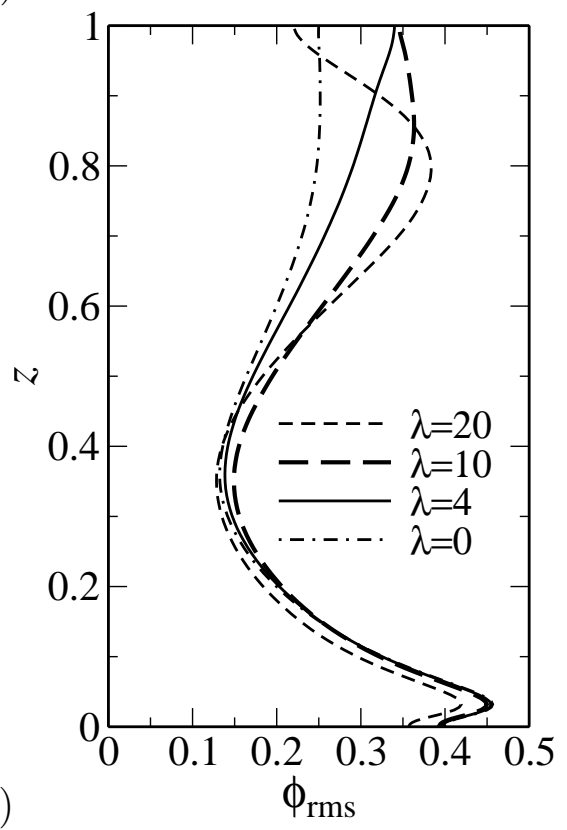


Figure 3: Contours of temperature $\phi-\phi_{0}$ in the $x z$ plane $(z$ is vertical and $x$ is horizontal) for (a) $\lambda=20$, (b) $\lambda=10$, and (c) $\lambda=4$ where $\phi_{0}=\langle\phi\rangle_{z=0}$.

(a)

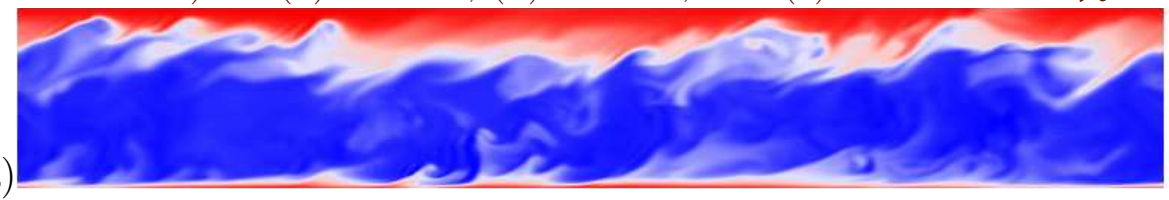
$-0.60$ $-0.10$ $-0.40$ $-0.90$ -1.4
-1.9

(b)
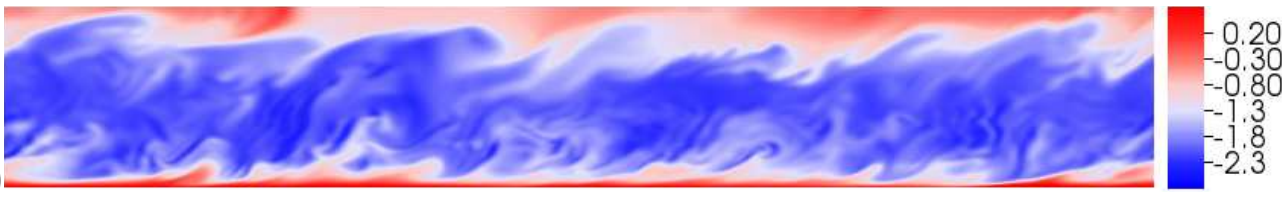

(c)

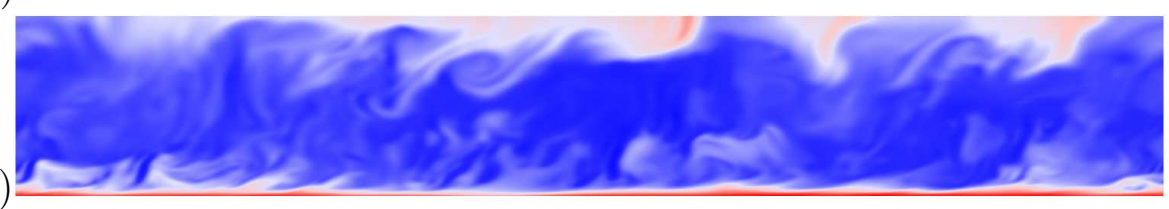

$-0.10$

$-2.4$

where the buoyancy flux $\mathrm{B}=-\lambda\left\langle\phi^{\prime} w^{\prime}\right\rangle$ and the turbulence shear production term SP $=-\left\langle u^{\prime} w^{\prime}\right\rangle d\langle u\rangle / d z$. Using Einstein notation for the velocity components $u_{i}$, the turbulent kinetic energy $k=0.5\left\langle u_{i}^{\prime} u_{i}^{\prime}\right\rangle$ and the turbulence dissipation rate $\varepsilon=1 / \operatorname{Re}_{\tau}\left\langle\left(\partial u_{i}^{\prime} / \partial x_{j}\right)^{2}\right\rangle$. The transport of turbulent kinetic energy by turbulent convection, $\mathrm{CT}=\mathrm{d}\left\langle w^{\prime} \mathrm{k}\right\rangle / \mathrm{d} z$. When the production and dissipation of turbulence is in balance, $\mathrm{SP}-\varepsilon-\mathrm{B}=0$. For $z \lesssim 0.36$, $\mathrm{B}>0$ and the total turbulence production $\mathrm{P}=\mathrm{B}+\mathrm{SP}$. However, for $\mathrm{B}<0$ the buoyancy flux contributes to the dissipation of turbulence so the total dissipation term $\mathrm{D}=-\varepsilon-\mathrm{B}$. The scaled turbulence transport terms are plotted in Figure 5, scaled by the total local dissipation D.

Figure 4 shows a two layer structure which characterises the SCBL. In the convective region $(z \lesssim 0.36)$, with increasing $\lambda$ the transport term $\mathrm{CT}$ is a significant source of turbulence, accounting for almost half of the local turbulence dissipation of $\lambda=20$ at $z=0.3$. At $\lambda=0$, the flow is in near local energetic equilibrium with $\mathrm{CT} \simeq 0$. The stable region $(z \gtrsim 0.36)$ has the opposite response to increasing $\lambda$. As the outer boundary layer becomes more 
Figure 4: Variations over the vertical location $z$ of (a) the mean vertical shear stress $\left\langle u^{\prime} v^{\prime}\right\rangle$; (b) the streamwise normal stress $u_{\text {rms }}$; and (c) the streamwise velocity profile.

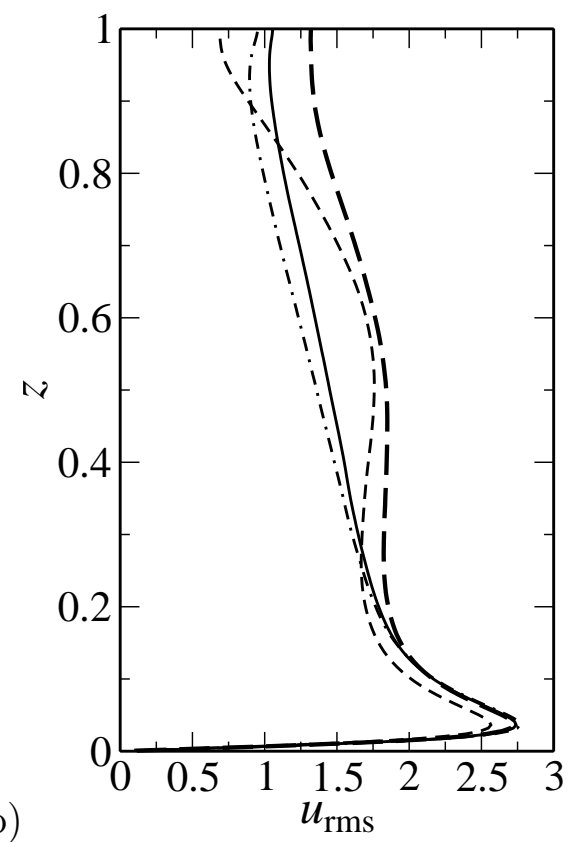

(a)
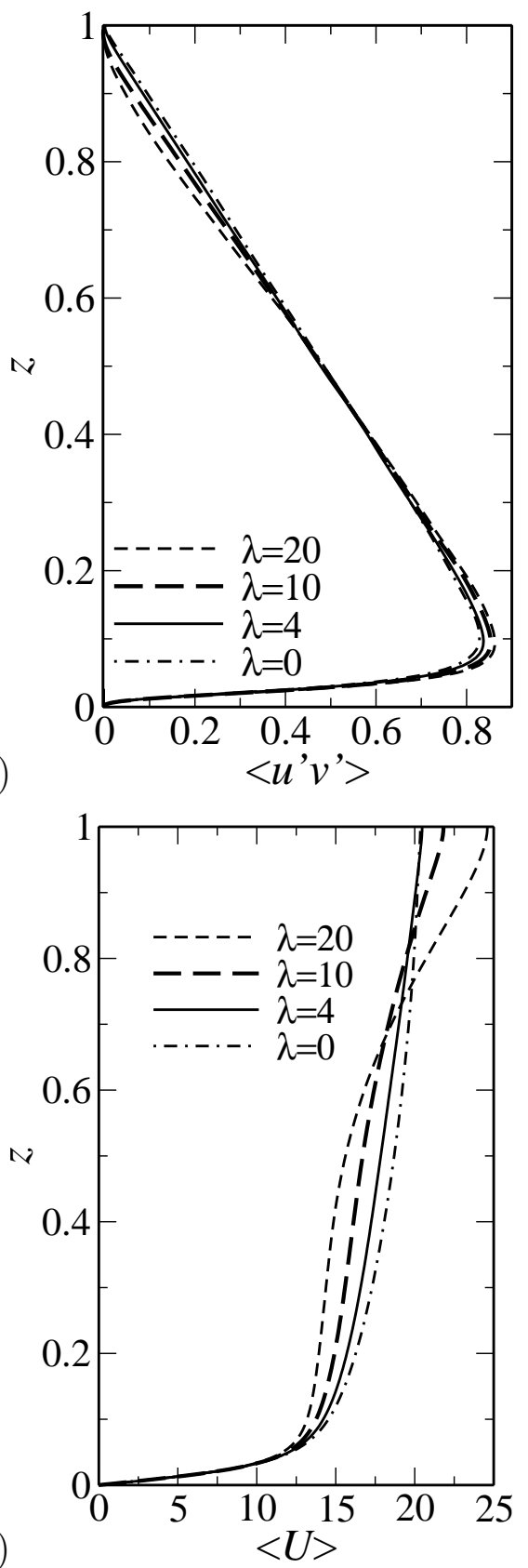
Figure 5: Scaled turbulence kinetic energy budget terms scaled by total turbulence dissipation $\mathrm{D}=-\varepsilon-\mathrm{B}$ : (a) turbulence shear production SP; (b) buoyancy B; and (c) convective transport term CT.

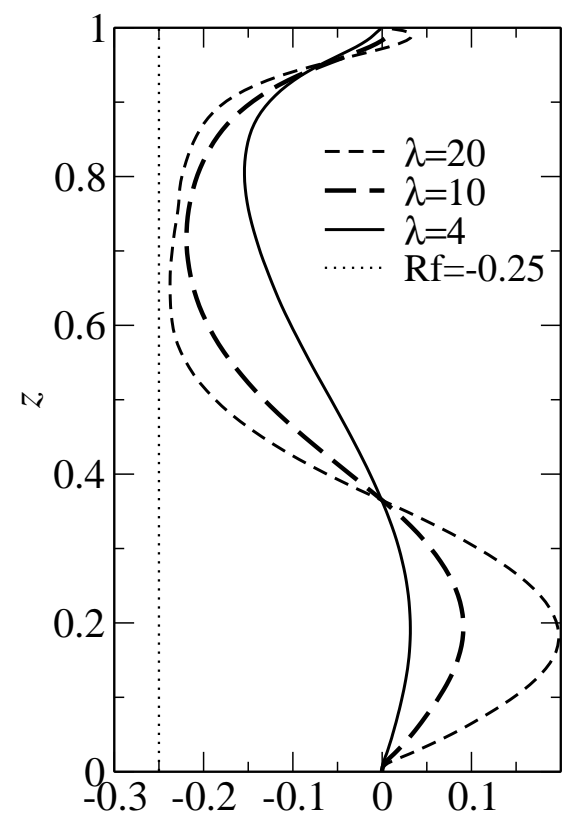

(b) $\mathrm{B} / \mathrm{D}$ (a)
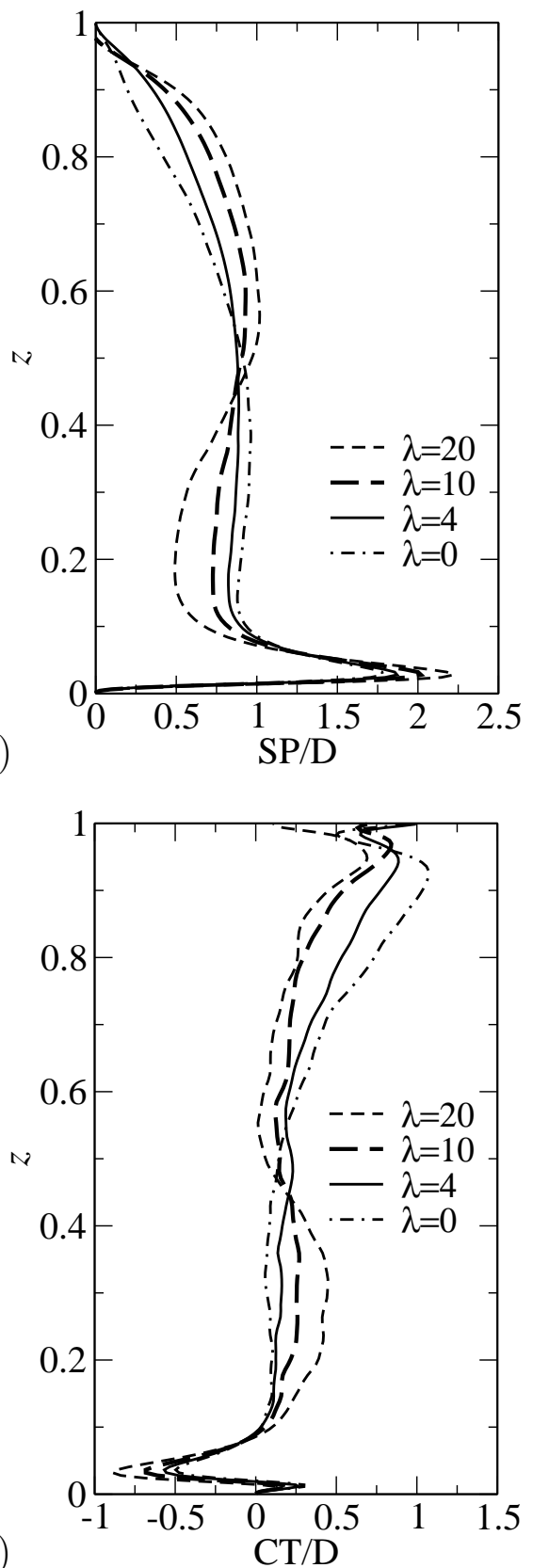
Figure 6: Gradient Richardson number with $\mathrm{Ri}=0.25$ indicated.

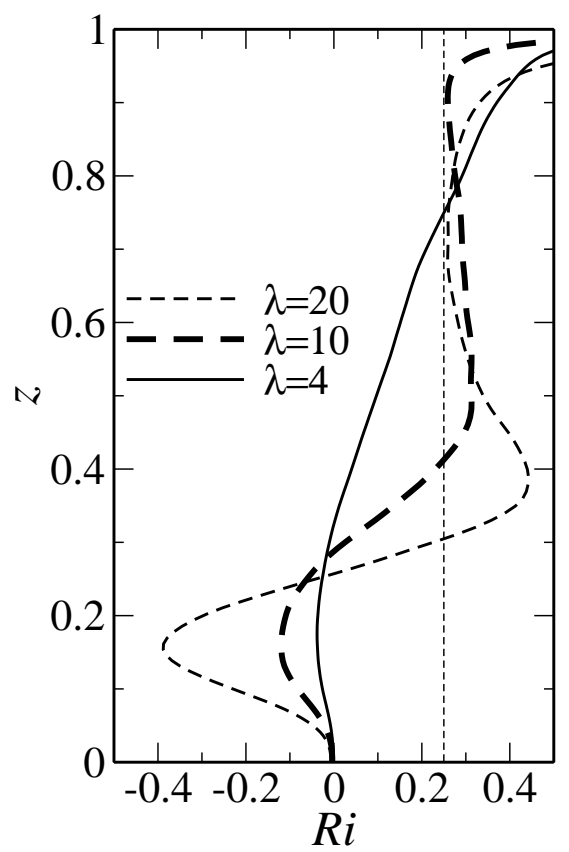

stable, the local shear production term becomes an increasing proportion of the total turbulent energy dissipation with local equilibrium achieved over approximately $0.5<z<0.7$. At $\lambda=0$ the transport term $\mathrm{CT}$ is a significant source of turbulence, accounting for more than half of the local turbulence dissipation.

The scaled buoyancy term B/D is also known as the flux Richardson number $R_{f}$. In stably stratified shear flows limit values of $R_{f}=0.25$ were widely observed [3]. In the present results, Figure 5(b) shows that for $\lambda \geqslant 10$ the flux Richardson number approaches the limit of $R_{f}=B / D=0.25$ over $0.5 \lesssim z \lesssim 0.8$. Similar limits are observed in the gradient Richardson number, $\mathrm{Ri}=\mathrm{N}^{2} / \mathrm{S}^{2}$ where $\mathrm{N}^{2}=-\lambda \mathrm{d}\langle\phi\rangle / \mathrm{d} z$ and $\mathrm{S}=\mathrm{d}\langle\mathrm{u}\rangle / \mathrm{d} z$. In Figure 6 $\mathrm{Ri}=0.25$ in the stable shear layer. In the SCBL both limits of these values were observed [6]. 


\section{Conclusions}

The transition from neutral to strongly stratified flow in a thermally stratified open channel flow was examined using direct numerical simulations. The flow has both a stably stratified surface layer and a unstably stratified near wall region. The flow attained is shown to have similar character to the shear convective atmospheric boundary layer.

In the stable thermocline for $\lambda=20$, both the flux Richardson number and gradient Richardson number approach limit values $R_{f}=0.25$ and $R i=0.25$, respectively, similar to limits seen in other stably stratified shear flows. The turbulence transport term is small so the flow is near local energetic equilibrium over most of the stable shear layer $0.5<z<0.8$, which is expected to aid turbulence parameterisations. At $\lambda=20$ and $\alpha \delta=1.5936$ turbulence is strongly damped near the free surface.

The most interesting feature of the flow is that buoyancy contributes significantly to turbulence production in the near wall region but it is unclear how the convective flow contributes to the shear that develops in the outer boundary layer, if at all. This will be the focus of future work.

Acknowledgements: The authors gratefully acknowledge the support of the Australian Research Council (ARC). The first author was supported by ARC Post-doctoral Research Fellowship DP110103417.

\section{References}

[1] S. M. Mitrovic, L. Hardwick, and F. Dorani. Use of flow management to mitigate cyanobacterial blooms in the Lower Darling River, Australia.

J. Plankton Res., 33(2):229-241, 2011. doi:10.1093/plankt/fbq094 C247 
[2] J. R. Taylor, S. Sarkar, and V. Armenio. Large eddy simulation of stably stratified open channel flow. Phys. Fluids, 17(11):116602, 2005. doi:10.1063/1.2130747 C248

[3] M. Garcia-Villalba and J. C. del Alamo. Turbulence modification by stable stratification in channel flow. Phys. Fluids, 23(4):045104, 2011. doi:10.1063/1.3560359 C248, C258

[4] N. Williamson, S. W Armfield, M. P. Kirkpatrick, and S Norris. A canonical model for stratified flow in estuaries and rivers. ANZIAM J., 54:C88-C101, 2012. http://journal.austms.org.au/ojs/index.php/ ANZIAMJ/article/view/6429 C248, C250

[5] S. W. Armfield, S. E. Norris, P. Morgan, and R. Street. A parallel non-staggered Navier-Stokes solver implemented on a workstation cluster. In S. Armfield, P. Morgan, and K. Srinvas (Eds.) Computational Fluid Dynamics 2002: Proceedings of the Second International Conference on Computational Fluid Dynamics, Sydney, Australia, 15-19 July 2002, pages 30-45. Springer, 2003. doi:10.1007/978-3-642-59334-5 C252

[6] R. J. Conzemius and E. Fedorovich. Dynamics of Sheared Convective Boundary Layer Entrainment. Part I: Methodological Background and Large-Eddy Simulations. J. Atmos. Sci., 63(4):1151-1178, 2006. doi:10.1175/JAS3691.1 C253, C258

\section{Author addresses}

1. N. Williamson, School of Aerospace, Mechanical and Mechatronic Engineering, University of Sydney, NSW 2006, Australia. mailto:nicholas. williamson@sydney .edu.au

2. M. P. Kirkpatrick, School of Aerospace, Mechanical and Mechatronic Engineering, University of Sydney, NSW 2006, Australia. 
3. S. W. Armfield, School of Aerospace, Mechanical and Mechatronic Engineering, University of Sydney, NSW 2006, Australia.

4. S. E. Norris, Department of Mechanical Engineering, University of Auckland, Auckland, New Zealand. 